\title{
Application of a neuro-fuzzy model to landslide-susceptibility mapping for shallow landslides in a tropical hilly area.
}

\begin{abstract}
This paper presents landslide-susceptibility mapping using an adaptive neuro-fuzzy inference system (ANFIS) using a geographic information system (GIS) environment. In the first stage, landslide locations from the study area were identified by interpreting aerial photographs and supported by an extensive field survey. In the second stage, landslide-related conditioning factors such as altitude, slope angle, plan curvature, distance to drainage, distance to road, soil texture and stream power index (SPI) were extracted from the topographic and soil maps. Then, landslide-susceptible areas were analyzed by the ANFIS approach and mapped using landslide-conditioning factors. In particular, various membership functions (MFs) were applied for the landslide-susceptibility mapping and their results were compared with the field-verified landslide locations. Additionally, the receiver operating characteristics (ROC) curve for all landslide susceptibility maps were drawn and the areas under curve values were calculated. The ROC curve technique is based on the plotting of model sensitivity - true positive fraction values calculated for different threshold values, versus model specificity true negative fraction values, on a graph. Landslide test locations that were not used during the ANFIS modeling purpose were used to validate the landslide susceptibility maps. The validation results revealed that the susceptibility maps constructed by the ANFIS predictive models using triangular, trapezoidal, generalized bell and polynomial MFs produced reasonable results $(84.39 \%)$, which can be used for preliminary land-use planning. Finally, the authors concluded that ANFIS is a very useful and an effective tool in regional landslide susceptibility assessment.
\end{abstract}

Keyword: GIS; Remote sensing; Landslide; ANFIS; Neuro-fuzzy. 